\title{
SOBRE LA PENETRACIÓN DE HISPANISMOS EN NAPOLITANO E ITALIANO
}

Tras la muy meritoria, aunque farragosa, recolección de materiales sobre elementos españoles en italiano llevada a cabo por Zaccaria ${ }^{1}$, contamos ahora con el valioso trabajo sistemático de Beccaria sobre reflcjos hispánicos en la lengua italiana de los siglos Xvi y $\mathrm{XVII}^{2}$. $Y$ en fecha más reciente ha aparecido el estudio consagrado a los clementos españoles del napolitano de I'. d' $\Lambda$ scoli, utilisimo, pero de muy difercute intención científica y metúdica ${ }^{3}$. Con todo, no obstante los méritos de cstos trabajos y la minuciosa atención que presta Beccaria a los clialectos mericlionales, incluido el napolitano, juzgamos de interés precisar

$\because$ ci proceso y el grado de penctiarión de los hisjunismos en el napolíano $y$ en la lengua nacional. Para poder efectuar tal indagación es mencster, naturalmente, en relación con los documnitos y los hechos, tener ante todo presente, entre los dialectoss meridioneles, al siciliano, donde el influjo español tuvo iguainente nucha iminotancia, para ter si los hisplanismos penetraron en italiano efectivanente a través del napolitano. Asimismo, es muy importante fijar a través de que ambionte los hispanismos chtraron en el diapolitaito y el íntiano. Daste ei punto de vista cronológico, hemos de tener en cuenta los hispanismos que cutran en el napolitano durante el grubierno de los virreyes españoles ('nl los siglos xvi y xvil o, anteriormente, durante el reinado de los soberanos aragoneses, es decir, en e1 llamado período aragoniés.

1 L. Zaccaria: Raccolta di voci affatto sconosciule o mal hotc ai lessicografi ed ai filologi. MIarradi, IgI9, L'Elcmento iberico szlla lingua ilaliana. Bolonia. 1921. Iistos dos estudios son los más conocidos de Zaccaria entre otios diversos trabajos suyos sobre el mismo asunto.

- G. I. I3riccaria: Spagnolo e spagioli in Italia. Riflessi ispanici sulla lingua ilabiana del Cinque e del Seiccnto. 'Turfin, rg68.

- I. I'Ascor.I: Lingua spagmeola $c$ dialetlo napoletano. Con un discorso inirodullivo de $A$. Ariramura. Nápolcs, 1972. 
Por lo que se refiere al ambiente, tomemos primero algunos ejemplos de hispanismos en la lengua administrativa y de cancillería de la Nápoles de los siglos XVI y XVIr. En cierta petición dirigida al soberano español Felipe II, rey de Nápoles y Sicilia, por un gentilhombre napolitano Giovanni Donato della Marra, en I557, a propósito de los famosos useggi napoletani -centros de culace entre la administración de la ciudad y la baronia ${ }^{2}$ - encontramos hispanismos crudos $^{2}$, como por ej. cada giorno en lugar de ogni giorno ${ }^{3}$ o hispanismos no crudos, por ej. napol. accapà (< esp. acabar 'terninar, llevar a cabo, obtener, conseguir') 4. Se trata aquí, pucs, de un texto del siglo xv, redactado en italiano por un gentilhombre napolitano en un ambiente administrativo y cancilleresco, que permite ver si no cierto bilingüismo, al menos una estrecha convivencia entre napolitanos y españoles ${ }^{6}$. Huelga decir que los hispanismos efímeros y crudos como "cada giorno", caracteristicos de cierta situación histórica, no tienen mucha importancia desde el punto de vista lingüístico.

Puede causaruos extrañeza que la referida petición del gentilhombre napolitano al soberano español esté datada en Brusclas. Para aclaración de este hecho, es menester poner de relieve que, en la administración de la soberanla española en Nápoles, los Países Bajos con Flandes y, naturalmente, Bruselas ${ }^{6}$ tuvieron un puesto importante: pensamos en la premática $D e$ officiorum provisione de $155^{\circ}$ y en los contemporáneos "capítulos de Brusclas", cuya revocación redundó en muchos casos en

1 R. VILIARI: La rivolta antispagnola a Napoli. Le origini (i 585-1647). Bari, 1967, p. 180.

2 Para los préstamos crudos vid. B. E. Vidos: Prestito, espansione e migrazione dci termini tecnici nelle lingue romanze e non romanze. Problemi, metodo e risultati. Biblioteca dell'Archivum Romanzicum, serie II, vol. 3I. Florencia, 1965 , pp. 5, 7, 24, n. I, 355, 369 y n. I.

3 "... clie già non saria stata gran cosa clie avessero le Signorie Vostre mandata una lettera di cambio a questi Cavalieri vostri confidenti, con ordine che, cspediti mici negozii, wi avcssero falto fermare qui con darmi cada giorno il vitto necessario.... (Archivio storico italiano), 1846, IX, p. 180.

- In Napoli ci ingannammo del negoziar di questa Corte, e del valore qua delle genti; e siatc certo, che si alcuna cosa s'accapasse, ne sard non altri che la nostra giustizia.... (Archivio storico ilaliano), 1846, IX, p. 177. Cfr. BECCARIA: o. c., pp. 265 n., $266 \mathrm{n}$.

- B. Croc1: La lingua spagnola in Italia. Roma, r895, pp. I4-15.

- Para la importancia de Bélgica en la administración de la lacienda pública del gobicrno español en los Paises Bajos, vid. B. E. Vmos: Relaciones antiguas entre España y los Paises Bajos y problemas de los prestamos holandeses (flamencos) en el castellano, en RFE, 1972, LV, pp. 237-238. 
provecho de los napolitanos ${ }^{1}$. Conviene tener presente que, dusante la doninación española, las relaciones entre Nápoles y los Países Bajos (Flandes, comprendido el territorio francés-valón de Bélgica, y Holanda) eran muy estrechas, y que gran número de soldados napolitanos tomaron parte en las filas del ejército español en las guerras de Flandes ${ }^{2}$. Notable cra también la aportación de la aristocracia napolitania a las dichas guerras de España en Flandes ${ }^{3}$. En el estudio de las relacioncs existentes en los siglos xVI y xvin entre Nápoles y los Países Bajos, hay (ue scñalar la presencia en Nápoles de soldados valones alistadus en el cjército español y su insoleucia para con las damas napolitanos ${ }^{4}$. así cono los amotinamicntos de las tropas españolas en Iilandes ${ }^{5}$ en ticmpos en que, entre dichas tropas, se contaban fucrzas procedentes de Nápoles, encuadradas en el ejército español que coinbatía en Ilolanda ${ }^{\circ}$.

Para ilustrar estas relaciones entre Nápoles y los Países Eajos iajo dominio español, tomemos ante todo un término pertencicinte al an1bicnte de lenguaje cancilleresco: el it. cntregare del csp. cntregar ( $<$ irl-

1 VH,IARI: O. C., PP. 20-21.

2 VHILAkI: O. C., p. 82 .

3. Croct: Storia del regno di Napoli. Bari, 1925, pp. I05-111; VILI.ARI: o. c., p. 239 .

- "I"̈avviso, che per tutti li luoghi ove sono alloggianenti di soldati V'ailoni, per le loro insolenze ne siamo stati aumazzati molti... Questi Valloni, che vammo in alloggiamento, fanmo gran male, se S. Li. non ci rimedia; poichè vanno pigliatido le domne per forza, come hanno fatto ad un altro, oitre il sopradetto medico; e questo faltoli con li parenti un imboscalta, ne have ammaztati circlue, insine con la domnan (r6r8), en las Narrazioni tratle dai giornali del goierno di Don Pistro Girone Duca d'Ossuna vicerè di Napoli scritti da Francesco Zazzera (I6I6-1620) (Archivic storico italiano (1846). IX, p. 535).

- "Iil desconcierto llega al colno a la muerte de IRequeseus; los Fistados declaran relucldes a los soldados cspañoles amotinados en Alost [población de Flatides, "n flamenco Aalst]..." "... en atención a los desórdenes cometidos por los anotinados de Alost..." "Iodo esto no iupidió el que los amotinados de Alost, quemaran algtuias aldcas...». (L. DE TORRE: Los motines militares en Flandes, (n Rcvista de Archivos, Bibliotecas y Museos (I9I I), XXV, p. 227, XXVII (1913). pp. $370,372-373$.

- Temian los jefes que estallara el motín poco después de la batalla, y, para cvitarlo, enviaron al tercio de Nápoles al otro lado del río, al arrabal de Grave [población de Holanda].... Vale la pena citar a este propósito las palabras del Duque de Alba al rey Felipe II: "Todos los tercios se sublevan, hasta el de Nápoles..... (I. DI: Torre: o. c. en Revista de Archivos, Bibliolecas y Museos (1913), XXVI, p. II3 (IgII), XXV, p. 226. 
tegrare) ${ }^{1}$, hispanismo aislado y efímero en escritos napolitanos no literarios de fines del XVI y del XVII ${ }^{2}$. Nos parece interesante que, siendo éste un término militar de administración y cancilleria de la ocupación española, encontremos en los Países Bajos, entonces también bajo dominio cspañol, el hispanismo fr. entreger en una relación militar de I $334^{3}$.

Si encontramos, pues, un hispanismo en la lengua administrativocancilleresca de las secretarías italo-napolitanas del siglo XvIr que existe también en el francés de los Países Bajos y que ha dejado huellas en el italiano literario, podría sostenerse que el centro de difusión de tal hispanismo en Italia ha sido el napolitano. He aquf por ej. el it. alborot( $t$ )are 'sublevarse contra alguien', 'amotinarse', que se lee en una relación oficial hecha en Nápoles el año I636 en un ambiente español, que habla, entre otras cosas, de ciertos levantamientos en el palacio del Papa 4 . Teniendo presente la cronología y el tenor de los textos en donde la voz se halla, resulta claro que se trata de un término difundido en el siglo xvir en un vasto territorio bajo dominio español ${ }^{5}$, ya que se refiere a los amotinamientos en Europa. El it. alborot(t)are ${ }^{6}$

1 J. Corominas: Diccionario critico etimologico de la lengua castellana. BermaMadrid 1954-I957, II, p. 30r.

2 B. CROCre: La lingur., p. 55; BrecarLa: o. c., p. 39.

- "Et mena S. A. avecq soy 25 chariots chargez d'argent quil avoit de reste de son voyage, et comanda d'incorporer cest argent avecq l'argent du marquis, et ainsy fut enzirege à Edouard François Scortza, qui faict l'office de pagador gènéral en campagneu (Les Rélations militaires des annes 1634 et 1635 , rédigées par Jran-ANTOINLi Vrincar'r, Secrétaire des avis secrets de guerre aux Pays-Bas. Editées avec une introduction et des notes par M. HUISALAN, J. Drondr et I. VAN Merorberck. Académie Royale de Belgique. Commission royale d'histoire. Bruselas, I958, p. 88), vid. J. HIiRHLION: Elérnerts espagnols en wallon et dans le franfais des ancicus Pay's-Bas. Mémoires de la commission royale de toponymie et de dialectologic (section wallonne), Io. Lieja, I96r, p. 75.

- a... subito si parti un Padre di San Pietro a Maiclla Celestino a dar conto del seguito in Roma: che, sccondo l'avviso clie tiene S. I. dal signor Imbasciatore in Roma, alborotto la casa del Cardinal di Savoia ed il palazzo del I'apa...., en la Relazione diretta al Sig. Duca di Medina de las Torres intorno allo stato presente di varie cose del regno di Napoli, etc. (Archivio storico per le province napoletane, 1879, IV, p. 232, nota). Cfr. Crocr: La lingua., p. 56; BECCARIA, o. c., p. 34 .

- Cfr. por ej. en el sardo campid. alborot $(t) a i$ 'trastomar, desarreglar, perturbar' (frecuentemente aplicado con referencia a los trastornos de estómago), de procedencia española (M. L. WAGNER: Dizionario etimologico sardo. Heidelberg, 1960-62, I, p. 69).

- Ia forma albotorare por alborottare en C. BATTISTI-G. ALESsio: Dizionario etimologico italiano. Florencia, 1950-58, I, P. II2, debe de estar equivocada. 
se lee en el savonés Gabriello Chiabrera (I552-I638), al habiar de los españoles amotinados en Flandes (en la ciudad de Alosto [en flamenco Aalst]) y en Zelandia ${ }^{1}$. Después el it. alborotto 'commoción, agitacióil' figura en los Consulti mitedici de Francesco Redi (I626-I6gó) 2, tras io cual desaparece ${ }^{3}$. Assi pucs, el esp. alborots: 'irastornar, amotinarse', probablcmente de voliture ${ }^{4}$, se difunde con lus anutinaniculus de las tropas españolas ya en el siglo xvi en el francés de ios Países Lajos (alborote 'amotinamiento', alboroter 'amotinar' y los dcrivados alborolade, alboroteux $)^{5}$ y en siglo xvir en Italia, hablando de toda clase de conmoción, agitación, trastorno (por ej. de estómago) ${ }^{6}$. Así se explica que nuestro término aparezca casualmente en un texto napolitano de indole administrativo-cancilleresca y que, por lo demás, sea desconocido en napolitano ?. El centro de difusión, pues, de nuestro hispanismo no es el napolitano, por liaberse atestiguado anteriormente en el italiano literario y en el francés de los Países Bajos.

A continuación de los hispanismos llegados al napolitano a través del ambiente adninistrativo-cancilleresco en el periodo virreinal, examincmos una voz que se introdujo en Nápoles asimismo en ticmpos de los virreyes españoles, es decir en los dichos siglos xVI y xirl, pero no

1 Allora riaccuuistata Terisca in 7clanda, li Spignoli si alborolarono in Alosto; di donde venne cangione clie il Consiglio armasse incontra loron. "Disperse lc turbe alborotate di Fiandran (N. TOMMnSIO-13. BELIINI: Dizionario della lingua italiana. Turin, 1924, s. v. alborotare, alborolato, S. BATTAGLIA: Grande dizionario della lingua italiana. Turín, I96x y siguientes s. v. alboro(t)lare). Para los amotinamientos de las tropas españolas en Flandes, vid. mis abajo p. 67.

- M'inumagino che a prima vista questo rimedio metterà in alborotto; ina be io non lo credessi opportunissimo non lo avrei proposton (TOMMASEO-BeLIINT: o. c., s. v. alborotto: BATTAGLIA: o. c., s. v. alboròtto).

- ZaCCARIA: L'Elemento iberico., pp. I2-I3.

- Iil esp. alborotar, alboroto y los otros derivados son antigucs y se encuentran documertadisimos, vid. Diccionario ie Autoridades. Iradrid, 1726-1739, Diccionario histórico de la lengua española. Acadcuia Española. Miadrid, 1933-1936, Diccionario de la lengua española. Real Academia Iispañola, Madrid, I939, S. GU,I Gaya: Tesoro lexicográfico (I492-I726). Madrid, 1947; CoRonunas: o. c., I, pp. 88-89.

b hurililon: o. c., p. 48.

- Wagniar: o. c., I, p. 69.

7 El vocablo falta en F. GALIANI: Vocabolario delle parole del aialelto napoletano, che piu si scostano dal dialetto toscano, con alcune ricerche etimologiche sulle medesime degli Accademici Filopatridi (a cura di Farao-Mazzarella). Nápoles, 1789; 3. PUOTr: Vocabolario donestico mapoletano e toscano. Nápoles, I 841 ; V. DE RITIS: Vocubolario napoletano lessicografico e storico. Nápolcs, 1845-185I; R. D'AuBRA: Vocabolario napolitano-tosca:zo domestico di arti $c$ mestieri. Nápoles, 1873; A. A.-

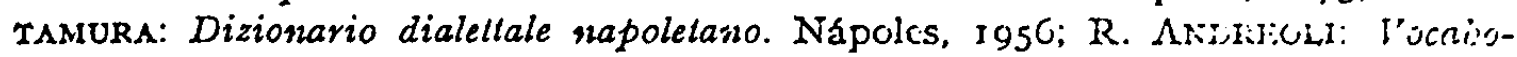
lario napoletano-italiano. Turin, I 887 (reimp. Náfolcs, Ig56). 
a través del ambiente cancilleresco-administrativo, sino por la vía de acceso de las cortes hispano-napolitanas, completamente promiscuas, en las cuales, por ejemplo, la guardia de honor del virrey Don Pedro de Toledo estaba compuesta de cien caballeros, la mitad españoles, lir otra mitird napolitanos: los "cien continos", de los que formaba parte c) conciilo poeta napolitano Iuigi Tansillo, elegido para enaltecer las cmpresas del virrcy ${ }^{1}$. Un lérmino típicamente cortesano es la voz napolitana insaio (siglo xvir), usada para designar la 'prueba de una obra tcatral para poncrla en el grado de perfección que le corresponde antes de su cstreno' o, en general, la 'prueba de cualquiér espectáculo para ponerlo a punto antes de presentarlo al público'. En este último sentido, encontramos usada la voz en las relaciones de los acontecimientos celcbrados en el palacio virreinal de Nápoles en los años I63r y I632, las cuales se refieren al insaio de ciertos brillantes espectáculos organizados por un privado del virrey ${ }^{2}$. A pesar de que en siciliano insayàrisi, insayamentu se documentan ya a principios del siglo xvI (en otro sentido, hien es verdad, que en napolitano) ${ }^{3}$, la voz napolitana insaio a causa

1 Crocr: La lingua., p. 1.5; F. Franmir: Il Cinquecento, on la Storia letteraria a'Jlalia. Milán (Vallasdi), s. a., p. 186; D'Ascorr: o. c., pp. 54-55.

2 a... intraprese [hallando del aludido privado del virrey] anco a dirigere clal suo palazzo due volte per intsaio due battaglie navali a vista di tutti..." Aggionta alli gionali di Scipione Guerra (1631-1632), en el Archivio storico per le province napoletane (1912), XXXVIT, p. 138. Aumo Donini I632... poiche in Palazzo vi sono state comedie, e giunte di daue più volte, dove si d ballato privatamente molto spesso, c di più un tornco sotto titolo di Inssaion, Governo di D. Fernando Afan de Ribera Enrriquez Duca $D^{\prime}$ Alcala, en el Arclivio storico per le province napoletanc (191 I), XXXVI, p. 77I, cfr.' BECCARIA: o. c., pp. 22-23. En el dialecto napolitano actual tencmos nzajo 'prueba, ensayo' (en los diccionarios dialectales napolitancs, recogido solo por AltranuRA: o. c., s. v., uzàjo: véase también F. D'AsCoI,I: o. c., p. Eo), cu el calalıés nsaja 'ensayar, experimentar, instruir' (G. RonL.Fs: Dizionario dicalctialc delle tre Calabrie. Halle-Milán, 1932-34, s. v. nsaja). Para la pérdida de la rocal inicial ítona, véase G. Rouliss: Grammatica storica della liysgua italiana e dei stcoi dialctti. Ionetica. Turin, 1966, p. I70 (cfr. napol. 'ntrdgne'intestinos', entràgnze 'vlsccras', esp. contrañas [AITAMURA: o. c., s. v.; Corommas: o. c., II, p. 303; Zacchria: Elem. iber., p. 165]). En el sardo log. y camp. tenemos insay'are, -ai 'adicstrar, instruir' y' 'probar' (un vestido). (WAGNER: o. c., s. v. insayare).

3 Sicil. insayàrisi 'meditor', 'similo', 'exerceo', insayameniu 'simulacrum', insay'ari lu passu 'vador, -aris', insayamentu di lu passu 'vadatio, -onis' (L. CRISTOFORO SCOBAR: Vocalualarintm nebrissense ex siciliensi sermone in latimum $L$. Christoforo Schobare Bcthico interprete traductum. Venezia, I519-1520, s. v.), sic. nzajari 'probar si una cosa va bien y de modo proporcionado o no', nzajata 'prucba de un vestido antes de quedar terminado' (A. TRAINA: Nucovo vocabolario siciliano-ilaliano. Palermo, 1868, s. v.). El pansiciliano nzajari 'probar una prenda 
del scntido especial que tiene cn el ambiente de la coite, desconocido cn cl siciliano, viene seguramente del esp. cnsayo (< exagium)l que tiene desde antiguo el sentido especial de 'prueba de representación teatral', 'prucba de torneo' ${ }^{2}$, que corresponde exactanente al comprobado en las cortes hispano-napolitanas. El napol. insaio no ha pasado, sin embargo, al italiano.

IIe aruui, en cambio, una voz napolitania de procedencia española que con certeza pasa al italiano: el napol. acciuvaccio 'azabache' ${ }^{3}$, que se lec ya en $\mathrm{I}_{593}$ en un inventario napolitano de ropas y alhajas 4 . El vocablo, aunque solamente figure en el diccionario de Altamura - falta en los más corrientes diccionarios napolitanos ${ }^{5}$ - tenía que cstar en uso en el napolitano de los siglos xVI y xvir según se desprende del incompleto pero precioso diccionario de De Ritis ${ }^{\circ}$, que lo registra, por ej. en $1635^{\text {en el }}$ Pentamerone de Giambattista Basile $(1575-1632)^{7}$ y en el poena en

de vestir para ver si sienta bien', 'probar un vestido que está siendo confeccionado'. Agradezco vivamente a mi colega el prof. G. 'Tropea los datos que ha tenido la nuabiliclad de proporcionarne relativos al siciliano (vid. más arriba sic. infanti).

1 Corominas: o. c., II, p. 299.

- Fisp. ensayo 'lo mismo que Ensayc y prueba: como el de una Comedia, torneo u otro festcjo' (Diccionario de Auloridades, s. v.). '... la prueba que se haze de algún acto publico, quaudo primero se prueba cn secreto, cono ensayo de tornco o otro exercicio de armas. Ensayo, entre los comediantes, la prueba que hazen autes de salir al teatro' (Covarrubias (I6II), citado por Gili Gaya: o. c., s. v. ensajo).

3 AitTAMURA: o. c., s. v. acciavaccio.

- 'Un branchiglio sen vasetto d'acciavaccio guarnito d'oro con tre cateniglie conl il piede et dui giri d'oro smaltati' (R. BerTRE: Vestimenti e gioielli in aso nclle province napoletane dal XII al XVI secolo, en el Archivio storico per le province napoletane (1897), XXII, p. 33I). Cfr. BeCCARIA: o. c., p. 74.

- IValta en los diccionarios napolitanos ya citados de Galiaui, Puoti, D'Ambra c Andrcoli.

- Dr: RrTis: o. c., s. v. acciavaccio ndinota uma pietra di color bruno e che prende un bel lucido nel lavorarla. Nella Galizia sen facevano divense immagini pei pellegriui». Èn efecto $y$, como consecuencia de ello, según es bien sabido, en Santiago de Compostela una de las fachadas de la catedral sigue siendo llamada Fachada de la Azabacheria: "La fachada Norte de la Catedral, contigua al I'alacio Eipiscopal, lleva aquel uombre debido al de la calle en que estuvicron instaladas las tiendas de los azabacheros, fabricantes de las hoy escasisimas figuras que representan al Apóstol y a la Virgen (R. I. Iópez: Santiago de Compostela. Guía del Peregrino y del Turista. Santiago, 1943, p. 60). Sobre la industria de la azabacheria, véase el estudio de J. FrRRÁNDIz: Marfiles y azabaches españoles. Barcelona-Buenos Aires, 1928, Colección Labor, sección IV. Artes plásticas, N. ${ }^{\circ}$ 59-160, pp. 227-264.

- "La schiava...ncopp'a cchill" arvolo pareva na statola d'acciavaccio drinto na casa de sucraudon (Pentamerone, V, 9). 
cuatro cantos escritos en octavas reales La Mezzacanna, que fue compuesto en I669 por Giambattista Valentini ', igualmente en dialecto napolitano. Ia palabra napolitana viene del esp. azabache, de igual sentido, palabra usada desde antiguo ininterrumpidamente ${ }^{2}$, procedente del hispano-árabe zabí $g^{3}$. In italiano se lee un solo ejemplo de acciavaccio 'clase de antracita' en el precursor de la botánica científica moderna, uno de los más importantes precursores de Limneo, Federico Cesi (I585I630) * A causa de la forma que se ajusta perfectamente con la del napolitano, en donde la voz era antigua y gozaba de indudable difusión, el it. acciavaccio viene del napolitano y no directamente del esp. azabachc. Se trata de un término científico, del cual, en el precario estado actual de la lexicografía italiana, sólo se ha encontrado un ejemplo hasta hoy ${ }^{6}$.

1 E no nce vedarraie autro a la mmane

Ch'aniclle d'acciavaccio o pure d'osse,

O cierte vretille, e passi vosse (La Mezzacanna, I, ro2).

$\Delta$ e complazco en expresar mi vivo agradecimiento a mi colega el prof. G. Alessio por la ayuda que me ha prestado en relación con la cita que del poema La Mezzacanna hace De Ritis.

2 Diccionario de Autoridades, s. v. azabache, GIII GAYs: o. c., s. v. azabache, azabachero, azabachado. Diccionario historico de la lengua española. Academia Es. pañola, s. v. azabache, azabachino, azabaja, azabaje, Diccionario de la lengua espaiiola. Real Academia Española, s. v. azabache 'variedad de lignito, bastante dura y compacta, de hermoso color negro de ébano, y susceptible de pulimento. Úsase para hacer botones, dijes y otras obras de adomo:

3 Corominas: o. c., I, p. 314 .

- Iro reduti i nascimenti del gagate, anchorché lontano dal Gange, dell'actite ad csso prcssimo; nel che venga lodato Plinio, e liberato dalla calunnia, che comuncmentce se l'è data, d'averli insieme congilinti; similmente la natura

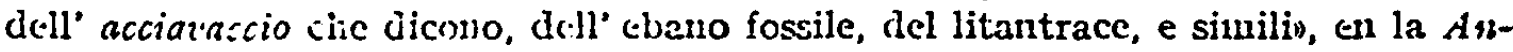
tolegia árlla prosa scientifica iacliana ciel Seicento, a cura di E. Fargur. Florencia, 1943 (listrisigin: o. c., s. v. aciicuaccio).

5 It. acciazaccio 'nombre de un pez', 'nombre de un pájaro' (no, en cambio, con c' significado de 'unat especic de antracita') aparece registrado en BATrISTrilusssio: o. c., I, p. 39. Hil sirnilficaclo de 'pc\%' io encontraron estos autores en la p. $25 S$ de !a insegura Raccolta cic Zaccaria, cque lo documenta en el navegante Gcmolli Cariti (1651-1725). Jictisti-Alessio ponen la voz en relación con el esp. azabache 'pájaro', que, sin embargo, no tiene el sentido de 'pez' (Diccionario de la Academia española, s. v. azabache 'pájaro de mos ocho centínetros de largo, con el lowo de color cenicicnto obscuro, el vientre blanco y la cabeza y las alas negras'). Fil que se le haya diıdo el nombre de 'azabache' a dicho pájaro en español, obedece, probablemente, a su color negro, ya que en cspañol azabache suele emplearse para designar todo lo que es de color negro brillante, por ej. cabello, ojos (Diccicturio de Autoridades, s. v. azabache umetaphoricamente se le da este nombre a cualquier cosa en sumo grado negran, Diccionario histórico de la lengua es- 
Tras esta pequeña exposición de españolismos entrados en el napolitano durante el período virreinal (siglos Xvi y XVII), veamos ahora un testimonio lingüístico interesantísimo del último tiempo del gobierno de la casa de Aragón en Nápoles, a fines del siglo xv. Se trata de la voz infante, infanta 'hijo o hija de los reyes de España'. Según Corominas', cl vocablo infante se extcudió desde España, probablemente desde el catalán, a Italia ya en los siglos xin y xiv. Para el siglo xin, Corominas remite a cierto testinonio que se lee en un artículo del Archivio glotlologico italiano. En relación con este testimonio, Corominas se basa con toda probabilidad en Menénclez Pidal ${ }^{2}$, quien, por su parte, sosLicne que el uso de la voz infante como título real se extendió desde Isspaña al suelo italiano. El artículo en cuestión es el trabajo clásico de Canello sobre los alótropos italianos ${ }^{3}$. Canello, a propósito del it. infante (alótropo fante 'soldado de infantería', 'servidor'), que significa 'niño' y, además, 'el príncipe heredero de España', observa que "quest' uso spagnolesco è tra noi fino dal sec. xirn" y nos da (p. 359) una documentación -en latín, que no en italiano- tomada de la Historia Sicula del cronista frauciscano Nicolo Speciale, siciliano, que narra las vicisitudes que atraviesa el reino de Sicilia de I282 a I3374.

Corominas, basándose en Zaccaria ${ }^{5}$, documenta la voz italiana on el siglo XIV, usada por Giovanni Villani. En efecto, el cronista florentino Villani, hablando "Della progenic del Re d'Aragona» emplea la voz infante en el sentido de 'hijo del rey'. Después, en un momento dado,

pañola, s. v. azabache aDicese de lo que es negro brillante, especialmente del cabello o de los ojosn). Cfr. GIII GAYA: o. c., s. v. azabache "La cosa muy negra conparamos a él, y dezimos ser negra como un afavache (CovarRubias [ $16 \mathrm{I}$ I])".

1 Diccionario, II, p. 979.

2 R. MInNéndez PIDAL: La leyenda de los infantes de Lara. Madrid, 1934, p. 443.

- U. A. CANELI.O: Gli allótropi italiani, en Archivio glotlologico italiano (I874), III, pp. 285-419.

- Auno domini 1295 Bonifacius pontifex, Fredericum, tunc vocatum insfaniem, Iacoli regis fratrem, magnis jam sollicitatum pollicitis, Roman [sic] vocat». Observemos, al respecto, que en el más antiguo (de principios del siglo xrr) diccionario siciliano-latino de SCOBAR: 0 . c. (véase p. 7o, nota 3), infanti se halla sólo con cl significado de infarrs, -antis 'niño'. Posteriormente, en el siglo XVu, encontramos el sic. infanti, infanta 'hijo o hija del rey' en el Antico Anonimo (Vocabolario siciliano italiano. Ms. anónimo inédito del siglo xvil de la Biblioteca Municipal de Palermo). Iin el siglo xvun léese en el Dizionario siciliano italiano latino de Mrciuisr Drir, BoNo (1'alermo, 1751-54): infanta o infanti di Spagna adicesi la figlia o il figlio non primogenito del re di Spagna: infante di Spagnan. Actualwcnte cn siciliano infanti significa 'niũo que aún no sabe hablar: infante' (TRAINA: o. c., s. v. infanti). Vid. infra el sic. insaydrisi, etc., p. 70 , nota 3 .

- L'Elemento iberico, p. 233. 
deja la casa aragonesa para pasar al tema central de su obra: los hechos de Florencia ${ }^{1}$. Infante en la obra de G. Villani es, pues, el primer ejemplo en italiano de este españolisno. Se trata de un préstamo aislado y ocasional, un préstamo, diríase, libresco, que aparece en una relación histórica y que, por consiguiente, no nace de circunstancias vividas. La documentación siguicnte, que figura, 'n la primera mitad del siglo xv, cn un escrito del célebre estadista florentino Rinaldo degli Albizzi al ayuntamiento de Florencia ${ }^{2}$ nos parece demasiado oficial y es, por tanto, asimismo, de indole libresca.

En cambio, la documentación siguiente de la voz infante en el sentido de 'hija del fey' es testimonio de circunstancias históricas verdadera y personalmente vividas. Federico III, de la Casa de Aragón, rey de Nápoles de 1497 a I50r, había contrafdo matrimonio en I486 (tras enviudar en I482) con Isabella del Balzo, hija de Pirro Balzo, príncipe de Altamura. Cuando Isabella del Balzo llegó reina a Nápoles (en octubre de I497) y, al cabo de algunos meses (en febrero de I498) pudo

1 "Quelli della casa d'Aragona, non furono anticamente di lignaggio reale, ma furono grandi conti di Barzalona \& di Valenza, \& come dicemo adrieto, l'antico loro, cio fu il conte Amfus, che fu sconfitto, \& morto da Franceschi à hoste à Carcasciana al tempo del Re Filippo il Bomio Re di Francia, \& dicesi che anticamente que d'Aragona furono d'wno lignaggio col conte di Tolosa, el buon conte Ramondo di Prouenza, ma poi il buono conte Giamo, figliuolo del conte Amfus, \& padre, che fu del detto Iicro Re d'Aragona, di cui trattiamo, che prese Cicilia per sua prodezza \& ualore, \& prese sopra i Saracini di Spagna il reame d'Araona, \& uccise lo loro Re, \& del reame si corono, \& popolollo de suoi Catalani, \& tutto uno reame con la Catalogna, \& fu egli \& suoi heredi, confermati Re d'Araona per la santa chiesa Romana, \& poi appresso per simile modo conquisto \& prese sopra i Saraciui lo reame \& isola di Mraiolica \& di Minorica, \& per hauere pace co Franceschi, diede per moglie ia figliuola al Re Filippo, che fu del buono Re Luis di Francia, $\&$ in dote parte della signoria di Perpignano \& di Mompolicri, \& quando uenne à morte lo Infante suo primogenito, cioe Piero detto di sopra, lascio Re d'Araona, \& Giamo ii. suo figliuolo Re di Maiolica, onde poi sono discesi ualenti Re \& Signori, come inanzi faremo mentionare... Isasceremo di quello d'Araona \& della rubellatione, infino che lungo \& tempo uerrà di cio parlare, \& tornaremo à nostra materia de fatti di Firenze, raccontando in breue altre nouitadi noteuoli per lo uniurso mondo aduenute in questi tempin. (La prima parte delle histoire universali de suoi tempi di Gionan Villani Ciltadino fiorentino, nuouanente ristampata con Tauole necessarie e Posfille in margine delle cose notabili, falle per Remigio fiorentino. In Venetia. Ad instantia di Giunti di Fiorenza. MOLIX, libro séptimo, cap. IXXXVI, p. 213).

2. A questi di scrivemo alla Signoria vostra della presura fatta del mese di marzo, per dua galee dello Infanten, en las Commissioni di Rinaldo degli Albizzi per il Comune di Firenze dal 23 luglio 1399 al 21 febbraio I432 (BATIAGLIA: o. c., s. v. infante'). 
reunirse de nuevo con ella - tras una larga etapa de adversa fortunasu marido el rey Federico, en estos momentos en que ambos cónyuges creycron ya descansar firmes en un trono firme, surgió un poeta napolitano Ruggiero di Pazienza di Nardò, que, resumiendo el conjunto de moticias y juicios y fáciles obscrvacioues moralcs que circulaban por Nípules sobre cl acontecimiento, compuso un poema italinno en ocho cantos en el que narraba las peripecias sufridas por la reina Isabella y que tituló Lo Balzino. En dicho poema, escrito hacia I498, léese la voz infante en el sentido de 'hija del rey', hablando el autor de la familia real ${ }^{2}$.

Esta documentación de la voz italiana en Nápoles alrededor de $\mathrm{I}_{498}$, aun sicndo también aislada y ocasional, nace de circunstancias vividas y refleja la vida en la Nápoles de fines del siglo xv, durante los últimos años del reinado de los soberanos aragoneses. Hemos de señalar también, hacia el fin del siglo xv, la presencia de la voz en el Compendio delle Istorie del regno di Napoli, 242 de Pandolfo Collenuccio ${ }^{2}$. Sin embargo, no tenemos huella alguna de la voz infante en el dialecto napolitano ${ }^{3}$ $y$, a menos que se descubra prueba en contrario, en los textos napolitanos. La ausencia de esta voz "hispano sensu» en el dialecto napolitano se comprende perfectamente teniendo presente que el gobierno aragonés en Nápoles toca a su término a fines del siglo xv y que la voz pertenece a la casa real y a la más alta esfera de la nobleza. Lo mismo podemos decir de la voz napolitana ciantro 'cantor de capilla', atestiguada en 1840 en el Almanacco Reale delle Due Sicilie, iutroducida y conservada por los Borbones de Nápoles como dignidad de la corte,

(B. Croce: Isabella del Balzino regina di Napoli in un inedilo poema siricrono, en Archivio slorico per le province rapoletane (1 897), XXII, pp. 632-634; B. CROCE: La Spagna nella vita ilaliana durante la Rinascenza. Bari, 1922, p. 69.)

2 Irasciando a la guardia e governo di Napoli l'infante don Piero suo fratello... con l'armata fe'vela a la volta di Spagnan (Batraglia: o. c., s. v. infante ${ }^{2}$ ).

- Ira voz falta en los diccionarios napolitanos de Galiani, Puoti, De Ritis, D'Ambra, Altamura, Andreoli, ya citados. 
del esp. chantre', documentado a partir del siglo xIrr, a su vez de origen francés ${ }^{2}$ o directamente del francés chantre. El napol. ciantro, lo mismo que infante, es voz empleada solamente en la corte y en el alto circulo de la nobleza $y$, por consecuencia, no figura en los vocabularios napolitanos ${ }^{3}$.

La opinión de Corominas que piensa que la penetración de la voz desde Isspana a Italia, y, por consiguiente, también a Nápoles, se produjo probablemente desde dl catalán, no ne parece aceptable, dado que ya, desde tiempos renotísimos, se venía usando, de modo general, cu cspañol antiguto if(f)ante, tanto en el sentido de 'niño' como en el de 'hijo del rey' 4 . El uso de la palabra, que en este último sentido se encuentra documentado ya en el año 973, se perpetuó, porque en general las familias nobles la emplearon hasta el siglo XIr en el sentido de 'hijo de padre noble'. Ya en el siglo xrrr, e incluso antes, infante ( $\operatorname{con} n$ ) fue el único uso de la palabra ${ }^{5}$. Desde el punto de vista fonético el esp. antiguo ifante viene, por cl cambio de $n f$ en $f$, del latín vulgar ifante ${ }^{\circ}$. La voz ifante (infante), a causa de la conservación de $f$ que hubiera. debido cambiarse en $v$ (escrita $b)^{7}$ y de la $i^{8}$, debe ser, a fin de cucntas,

1 ZACCARIA: Raccolta., pp. 91, 116, 187, 3I5; ZACCARIA: L'Elemento iberico., p. III.

2 Corominas: o. c., II, p. 19. W. v. WARTBURG: FEW, II, I, p. 236.

- Zaccaria: L'Elemento iberico, p. III; F. D'Ascoli: o. c., p. 53.

- Cid, verso 269 irem ante vos yo e vuestras ffijas, iffantes son e de dias chicas» verso 1279 ula mugier de mio Cid e sus fijas las iffantes" verso 3420 "pora los ifautes de Navarra e de Aragón verso 3448 "los ifantes de Navarra e de Aragón (R. MENEndez PLDAL: Castar de Mio Cid. Madrid, r969, vol. III).

5 R. MIINENDEZ PIDAL: Castar de Mio Cid, vol. II, pp. 720-723; R. MIENENDEZ PIDAL: La leyenda de los Infantes de Lara, pp. 442-443.

- G. BAIST: Etymologien, in Zeitschrifl für romanische Philologie, 1882, VI, p. 434; W. MLiver-LüBKr: Grammatik der romanischin Sprachcn. Ireipzig, 189o, vol. I, pp. 408-409; J. PIRSON: Le latin des formules mérovingiennes et carolingiennes, in Romanische Forschursgen, In09, XXVI, p. 937; M. JEANNERT: La langue des tablcties d'extcration latizres. Neuchâtel, 1918, p. 52; I. PAur.I: Enffant, garçont, fille dans les langutes romanes. Essai de lexicologie comparte. Iund, 1919, p. 26.

- R. Misntendez PIDAs: Masmial de gramálica histórica española. Madrid. I952, p. I3I.

- Que acaso después de la caida de la $n$ hubiera podido alargarse, como por ejemplo ell mèsa de meisam (cfr. F. HANSSFN: Spas:ische Grammatik anf historischer Gruidlage. Halle, I910, p. 53 y CoRournas: o. c., II, p. 997). 
un cultismo ${ }^{1}$, lo que se explicá considerando el uso de la voz cula esfera más alta de la sociedad.

Tras la presencia de la voz it. infante en el siglo xIV, en la primera mitad del siglo XV y hacia el final del mismo siglo xv en Nápoles, se la documenta posteriormente, en la prinera parte del siglo XVI, en Ariosto, cu un pasaje referente a cosas españolas ${ }^{2}$, y asimismo por ej. cn İ.Guicciardini (I483-I540) ${ }^{3}$ y G. Vasari (I5I2-I574) y llega a ser común cn los siglos XVI y XVII $^{5}$ como consecuencia de la gran influencia española ${ }^{\circ}$. Por todo ello, contrariamente a lo que ocurre con la voz acciavaccio, no puede afirmarse con seguridad que infantc, infanta hayan cutrado en italiano a través de Nápoles.

En cuanto al sentido de la voz, los diccionarios de la Crusca y Tommasco-Bellini nos desorientan afirmando que en la primera documentación en el siglo XIV (Villani) la voz infante significa 'hijo primogénito del rey' y a partir del siglo xvr simplemente 'cualquiera de los hijos del rey nacidos tras el primogénito' ?. Y sería asimismo equivocado el afirmar (que, en la documentación de la voz en Nápoles, hacia r49S, infante significa 'hija primogénita' de la familia real aragoncsa. 'Tanto en la obra de Villani como en el documento de Nápoles, infanle si toma cl sentido de 'primogénito' es porque en ambos casos se añade este atributo ("... \& quando ucune a inorte lo Infante suo primogenito..." cn Villani; "La infanle cra la figlia a la Regina, Primogenita..." en el

1 Muinfindi: l'nus,: Mamual, p. 137, atrilmye, ch ciccto, cl cambio de ifante

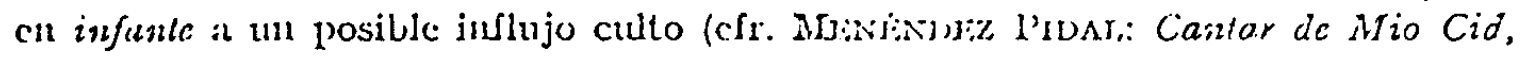
vol. I, p. 181).

- "Che, oltre i cavalicr", v'crano guide Che della bella infants avcano cura, Maturi vecchi, e assai clonne c donzelle Del regno di Gïanata, c le jiù bellen ARIOsro, Orlando furioso, I5, 5 I (Tommissio-Bieisini: o. c, s. v. infance).

3 "Delle galce catclane era cano lo infante, fratcllo dcl re Alfonso" GuiccluR1)INI, II-I80 (BATTAGLIA: o. C., S. V. inifante ${ }^{2}$ ).

- "ler INapoli ancora fece [IAca della Rolbhia] in Fioreuza la sepoltura di marno all' Infante frateilo del duca di Calarria, con molti ornamenti, aintato da Agustino suo fratcllo" G. VASARI: Vite dei piit eccellenti Pittori, etc., 3, 66 (Vocabolario degli accademici della Crusca. Florcncia, I863-1920, 5." imp:., s. v. infante).

- Crusca: o. c., s. v. infante c infanta, Tomusuo-Bitsint: o. c., s. v. infante, infantil, BAT'SAGLIA: o. c., s. v. infante e infanta.

- Is notorio que Ariosto lamenta la "vile adulazione spagnolan (F. Fr,Asuñ: Il Cinquccento, p. 20 en la Storia letteraria d'Italia. Nilán (Vallardi), s. a.

- Lista equivocación se lia corregido en el vol. VIIr, recicntenente aparccido. del diccionatio de BATraglis, s. v. infanta, doncie se lee que del titulo de infanta 'cra esclusa la primogenita a cui spettava il titolo di principessa'; véase uás abajo (p. 73, nota 4) el diccionatio siciliano de M. Dit, BONO (175I-54), que observa a propúsito de infanti: 'la figlia o il figlio non primogenito del re di Spagna'. 
documento de Nápoles) $y$, con precisión, se puntualiza que el infante - la infanta es primogénito o primogénita. En español infante, referido a la casa real, designa a todo hijo legítimo del rey nacido tras el principe - la princesa ${ }^{2} y$, en tiempos antiguos, fue llamado así también el primogénito del rey de Castilla añadiéndose «heredero" o "primero herederois ${ }^{2}$.

En suma, ya hemos podido ver, en lo que toca a este pequeño estudio, la importancia del ambiente en los préstamos y el interés del conocimiento de las circunstancias particulares de quién o de quiénes tomaron en préstamo una voz o emplearon tal voz (por ej. insaio e infante), importancia sobre la cual he insistido ya en otro lugar ${ }^{3}$. A propósito de la voz alborol(t)are, el ambiente cancilleresco-administrativo nos conduce desde Nápoles a los Países Bajos y nos hace ver que se trata de una voz si no internacional, al menos interespañola en el siglo xvr, usada, en la vasta monarquía española, por su administración tanto en Nápoles como en los Países Bajos.

Si para la voz acciavaccio no hubiéramos encontrado una docunentación italiana en el monumental diccionario en avanzado curso de publicación, pero todavía incompleto, de S. Battaglia, no habriamos por ej. podido afirmar que el esp. azabache penetró con seguridad a través del napolitano acciavaccio. El estudio fascinante de la penetración de hispanismos en Nápoles y en el italiano es harto arduo no sólo a causa del estado poco favorable de la lexicografía italiana, sino también porque exige investigaciones de los testimonios, podría decirse que oculares, que se encuentran especialmente en textos cuyo estudio no está tan adelantado como sería de desear ${ }^{4}$. En cuanto a las frases aisladas que figuran en los diccionarios, por lo común, no bastan, porque, a ser posible, se necesita ver el texto entero original para poder valorar el grado de penetración de los elementos extranjeros, según hemos podido comprobar en el caso de la voz infante.

B. E. VIDOS

2 Diccionario de la lengua española. Real Academia Española, s. v. infante, infarita 'cualquiera de los hijos varones y legitimos [las hijas legitimas] del rey, nacidos [nacidas] después del principe o de la princesa'.

- Diccionario de Autoridades, s. v. infante: 'Ahora se llaman assi los hijos del Rey, desde el segundo génito en adelante, y las hijas Infantas'. Diccionario de la lengua española. R. Acricmia Española, s. v. infante: 'Hasta los tiempos de don Juaı I se llanó asi también el hijo primogénito del rey. Se solfa añadir heredero, o primero heredcro'.

s Vidos: Prestito., pp. 247-268.

- Cfr. M. WIS, en Nerphilologische Mitteilungen, r969, LXX, p. 753. 